\title{
Magneto-optical light scattering from ferromagnetic surfaces
}

\author{
M. U. González, ${ }^{\text {a) }}$ G. Armelles, C. Martínez Boubeta, and A. Cebollada \\ Instituto de Microelectrónica de Madrid (CNM-CSIC), C/Isaac Newton, 8 (PTM), 28760-Tres Cantos, \\ Madrid, Spain
}

(Received 29 May 2002; accepted 21 November 2002)

\begin{abstract}
We have studied the optical and magneto-optical components of the light scattered by the surface of several Fe films with different morphologies. We present a method, based on the ratio between the optical and magneto-optical components of the scattered intensity, to discern the physical origin, either structural or magnetic corrugation, of the light scattered by these ferromagnetic surfaces. Surface versus bulk magnetic information can be separated by magneto-optical light scattering measurements, the scattered light being more sensitive to magnetization differences between surface and bulk than the reflected one. (C) 2003 American Institute of Physics. [DOI: 10.1063/1.1537511]
\end{abstract}

The magneto-optical Kerr effect has attracted much attention in the last years as a tool to probe the magnetic properties in thin films. Most of the studies extract information from the reflected beam, assigning the concluded properties to the film as a whole entity, and averaging the depth dependence. Nevertheless, lattice distortion or interdiffusion at the interfaces can give rise to strain and hybridization effects that can locally modify the magnetic properties of the film, such as a local magnetization reduction. Recent works studying the diffracted spots from periodic arrays of ferromagnetic structures ${ }^{1-3}$ show that magnetic information such as domain structure and magnetic inhomogeneities can be obtained. Following a different approach, analyzing the magnetooptical component of the scattered light can also be very useful to extract information on the magnetic behavior of surfaces and interfaces. Up to now, most of the work has been devoted to study the pure optical component of the scattered light, ${ }^{4-6}$ which offers information about surface morphology. Nevertheless, little is known about the modification of the intensity scattered by the surface of a ferromagnetic material when a magnetic field is applied. In this letter, we report our observations on the optical and magnetooptical component of the light scattered by a series of $\mathrm{Fe}$ samples, and we analyze the origin of the scattering mechanism and the kind of information that this technique could provide.

Single-crystalline $\mathrm{Fe}(001)$ layers (30 $\mathrm{nm}$ thick) were grown on $\mathrm{MgO}$ buffered GaAs substrates, ${ }^{7}$ with $2 \mathrm{~nm} \mathrm{MgO}$ or Pt capping layers to prevent oxidation. Atomic force microscopy (AFM) and angle-resolved light scattering measurements ${ }^{6}(\mathrm{He}-\mathrm{Ne}$ laser, $\lambda=633 \mathrm{~nm})$ were used to characterize the samples' surface roughness. The magnetooptical component of the scattered light was extracted using a modulated system that allows the detection of very low scattered intensities: A rotating magnetic field of enough intensity to saturate the sample is applied in the interface plane of the sample, thus forcing the rotation of the component of the sample magnetization in this plane. ${ }^{8}$ For a $p$-polarized incident beam, such rotation produces a variation of the intensity of the $p$-polarized reflected (scattered) light propor-

${ }^{a)}$ Electronic mail: ujue@imm.cnm.csic.es tional to the variation of the component of the magnetization perpendicular to the plane of incidence (transversal Kerr effect configuration). Therefore, the component of this $p$-polarized reflected or scattered beam at the frequency of the rotating magnetic field is the corresponding magnetooptical component, while the total intensity is the optical component.

In Fig. 1(a) we present the angular distribution of the optical component of the scattered light intensity $\left(I_{\mathrm{sc}}\right)$ in the plane of incidence for a sample with a rough surface. The surface morphology consists of a periodic one-dimensional corrugation oriented along one of the surface axes originating from the GaAs substrate. The period of the corrugation is $1.9 \mu \mathrm{m}$ and its rms roughness is $1.5 \mathrm{~nm}$. The surface also shows a smaller two-dimensional roughness with a rms value of $1 \mathrm{~nm}$. The curve with dots corresponds to the intensity scattered by the sample corrugation and presents two lobes [marked with arrows in Fig. 1(a)] associated with the corrugation period. ${ }^{6}$ The curve with squares corresponds to the light scattered by the disordered background roughness in the direction perpendicular to the corrugation, with no characteristic periodicity. Figure 1(b) shows the magneto-optical counterpart $\left(I_{\text {scmo }}\right)$ for these two azimuths. Differences be-

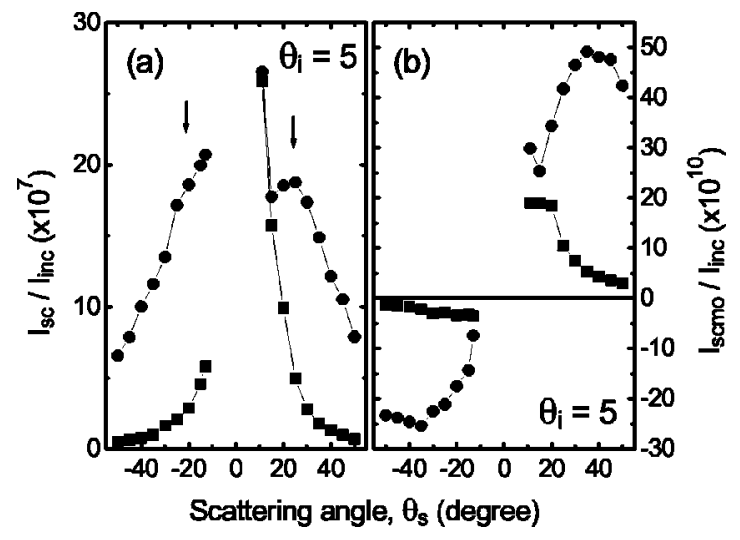

FIG. 1. (a) Distribution in the plane of incidence of the optical component of the light scattered by the corrugated surface of a ferromagnetic sample $\left(I_{\mathrm{sc}}\right)$ when the corrugation is perpendicular (dots) and parallel (squares) to the plane of incidence. (b) Distribution of the magneto-optical component of the light scattered by the same sample $\left(I_{\text {scmo }}\right)$ in the same azimuths. Both signals are normalized to the intensity of the incident beam $\left(I_{\mathrm{inc}}\right)$. 


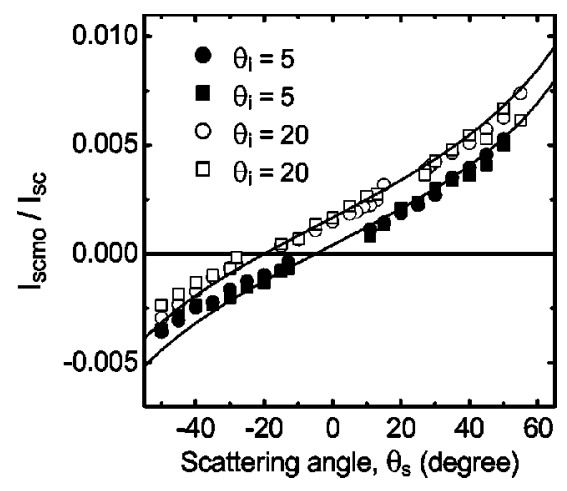

FIG. 2. Ratio between the optical and magneto-optical components of the light scattered by the surface of the same sample as in Fig. 1, for two angles of incidence, $\theta_{i}=5$ and 20. Discrete points represent the experimental data, with dots and squares corresponding to the measurements taken with the corrugation perpendicular and parallel to the plane of incidence, respectively. The lines are the theoretical calculations according to Eq. (1).

tween optical and magneto-optical components can be observed: For the magneto-optical part (dots), the lobe is located at a different angle than for the optical one, which, at a first glance, may suggest different periodicities for surface morphology and magnetization distributions. The $I_{\text {scmo }}$ measured perpendicular to the grooves (squares) shows a structure not seen in the corresponding optical component of Fig. 1(a). Moreover, $I_{\text {scmo }}$ reverses its sign for backscattering angles in both azimuths. However, despite the different surface structure present in the two azimuths, and the differences observed in the distribution between the optical [Fig. 1(a)] and magneto-optical components [Fig. 1(b)], their ratio, plotted in Fig. 2, is the same for both azimuths. As can be seen in Fig. 2, this ratio depends only on the angles of incidence and scattering, but not on the surface morphology.

To understand the observed behavior, we have calculated the intensity scattered by a generic ferromagnetic sample. Although scattered light could come from different sources, we assume here that surface roughness is the main mechanism of scattering. Then, if surface roughness is much smaller than the light wavelength, first-order perturbation theory can be applied. We have followed the method presented in Ref. 9, extended here to ferromagnetic materials (the problem geometry is shown in the inset of Fig. 3). The electromagnetic field in a medium $j, \mathbf{E}_{j}$, near the surface can

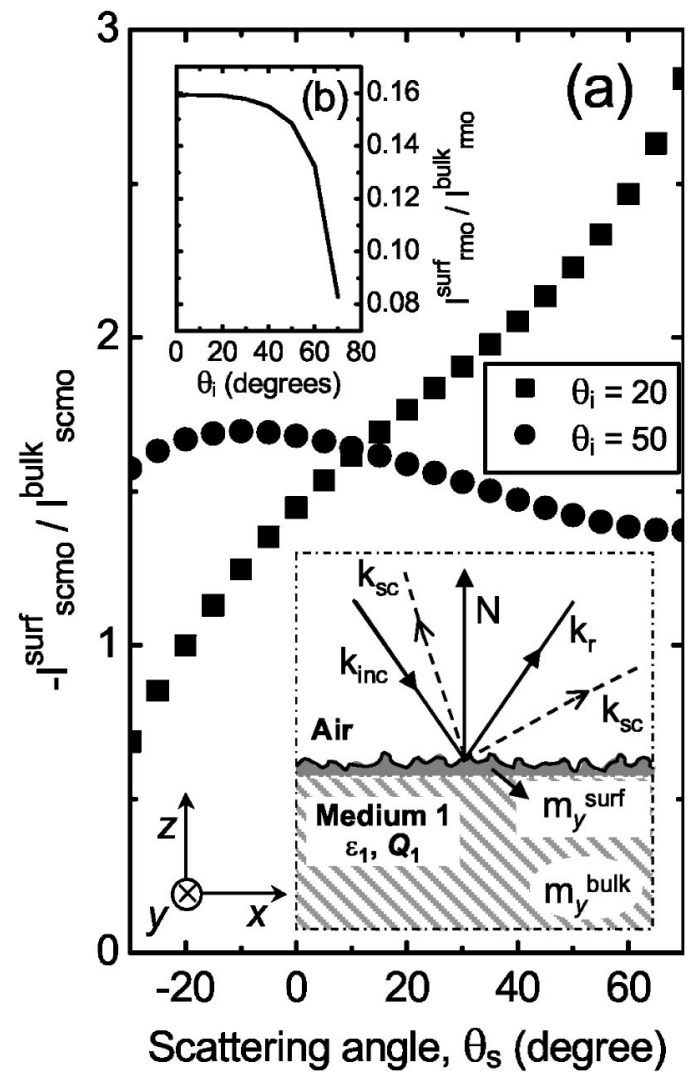

FIG. 3. (a) Ratio of the magneto-optical component of the scattered intensity coming from the surface layer and that coming from the substrate for a system where the magnetization of the surface layer $(1.5 \mathrm{~nm}$ thick) differs from that of the substrate (the inset shows a schematic representation of the problem). (b) Same as (a) for the reflected beam.

be expressed as a sum of a zeroth-order field (the field calculated for a perfectly smooth interface, $\mathbf{E}_{j}^{0}$ ) and a first-order correction due to the rough interface $\left(\mathbf{E}_{j}^{1}\right)$. The first-order field satisfies Maxwell equations in medium $j$, hence, its intensity can be easily calculated from the continuity of the total tangential component of the electric and magnetic fields at the interface. If the system is fully magnetized along the axis perpendicular to the plane of incidence ( $y$ axis), the intensity of the $p$-polarized light scattered into the air in the plane of incidence under $p$-polarized incident light is proportional to

$$
\left|\Delta Z\left(\mathbf{k}_{0}-\mathbf{k}\right)\right|^{2}\left|\frac{\varepsilon_{1}(1+T)\left(\sin \theta_{s}\right) \Delta E_{z}^{0}-\Delta D_{x}^{0}\left[(1-T) q_{s}-i(1+T) Q_{1} \sin \theta_{s}\right]}{(1+T)\left(\varepsilon_{1} \cos \theta_{s}-i Q_{1} \sin \theta_{s}\right)+q_{s}(1-T)}\right|^{2},
$$

where $\Delta E_{z}^{0}=E_{\mathrm{air}, z}^{0}-E_{1, z}^{0}$ and $\Delta D_{x}^{0}=D_{\mathrm{air}, x}^{0}-D_{1, x}^{0}$ are the discontinuities at a smooth interface (air/medium 1) of the $z$ and $x$ components of zeroth-order electric and displacement fields, respectively; $\theta_{s}$ is the scattering angle; $\Delta Z\left(\mathbf{k}_{0}-\mathbf{k}\right)$ is the in-plane spatial Fourier transform of the interface profile, being $\mathbf{k}_{0}$ (k) the projections of the incident (scattered) wave vector onto the $(x, y)$ plane; $q_{s}=\sqrt{\left(\varepsilon_{1}-\sin ^{2} \theta_{s}\right)} ; \varepsilon_{1}$ is the diagonal component of the dielectric constant tensor of medium 1 and $Q_{1}=-i \varepsilon_{r z}^{1} / \varepsilon_{1}$, with $\varepsilon_{x z}^{1}$ the nondiagonal comDownloaded 14 May 2010 to 161.111.235.252. Redistribution subject to AIP license or copyright; see http://apl.aip.org/apl/copyright.jsp ponent, which depends linearly on the magnetization in medium 1 along the $y$ axis, $\varepsilon_{x z}^{1}=B m_{y}^{1}$; and $T$ is the ratio of the scattered field at the interface in medium 1 induced by reflections in the layers underneath the surface layer and that propagating into the sample: $T$ can be approximated to zero if the optical properties of the surface layer and the layers underneath are very similar.

By using Eq. (1), we have calculated the Fourier transform of the surface profile from $I_{\mathrm{sc}}$ shown in Fig. 1(a), and 
the obtained function agrees completely with that extracted from AFM measurements, giving the same roughness characteristics (corrugation periodicity, rms roughness values), which indicates that this component of the scattered light is produced exclusively by the surface roughness for this sample, validating then this assumption in our analysis. On the other hand, Eq. (1) also shows that the ratio between the difference of scattered light intensities when the sample is fully magnetized along the positive and negative directions of the $y$ axis (proportional to $I_{\mathrm{scmo}}$ ) and the intensity of the scattered light when the sample is fully demagnetized (which can be equalled to $I_{\mathrm{sc}}$, since $I_{\text {scmo }}$ is three orders of magnitude lower, as shown in Fig. 1) does not depend on the roughness profile $(\Delta Z)$, as has been observed experimentally. $\Delta E_{z}^{0}, \Delta D_{x}^{0}$, and $T$ depend on the whole structure of the sample, and therefore, simple analytical expressions for this ratio can only be obtained for special cases. The full lines in Fig. 2 represent the theoretical calculation of this ratio, $I_{\mathrm{scmo}} / I_{\mathrm{sc}}$, for the sample shown in Fig. 1 . We have used the values of $\varepsilon_{x z}$ for the Fe layer given in Ref. 10, and the measured values of the complex refractive index. The very good agreement between theory and experiment strongly support the idea that the origin of both components of scattered light is the same: surface roughness. The different structures observed in the magneto-optical component of the intensity of the scattered light [Fig. 1(b)] are not due to differences between magnetic roughness and surface roughness, they are intrinsic to the angular distribution of the magnetooptical component of the scattered light.

Another relevant conclusion that can be extracted from Eq. (1) is that, with a purely optical technique and for roughness induced scattering, surface and bulk magnetic information can be separated, the scattered light being more sensitive to surface magnetization than the reflected beam. Let us consider, for example, a magnetically inhomogeneous system where the $y$ component of the surface layer magnetization differs from that of the underneath layer (substrate) (see the scheme in the inset of Fig. 3). In the transversal Kerr configuration, the intensity of the $p$-to- $p$ reflected beam $(r)$ or the $p$-to- $p$ scattered light (sc) can be expressed as $I_{\alpha}=I_{\alpha}^{0}$ $+I_{\alpha}^{\text {surf }} m_{y}^{\text {surf }}+I_{\alpha}^{\text {bulk }} m_{y}^{\text {bulk }}+O\left(m^{2}\right)(\alpha=r$ or sc $)$ with $m_{y}^{\text {surf }}$ and $m_{y}^{\text {bulk }}$ being the $y$ component of the magnetization of the surface layer and the substrate, respectively. Using a transfer matrix formalism, together with Eq. (1) for scattered light, we can calculate the ratio $I_{\alpha}^{\text {surf }} / I_{\alpha}^{\text {bulk }}$, which represents the sensitivity to surface magnetization. Figure 3 shows such a ratio for a system with a surface layer thickness of $1.5 \mathrm{~nm}$, assuming the same refractive index and magneto-optical constant (taken as those of $\mathrm{Fe}$ ) for surface and substrate. The ratio has been calculated for different angles of incidence, and in all cases the scattered light is more sensitive to surface magnetization than the reflected beam. Moreover, the sensitivity can also be tuned by changing the wavelength to probe particular surface layer transitions, therefore, increasing the sensitivity, in a similar way as done in x-ray resonant magnetic scattering. ${ }^{11-14}$

In conclusion, we have presented a method to determine the origin of the magneto-optical component of the light scattered by a ferromagnetic surface. We have shown that surface versus bulk magnetic information can be separated by magneto-optical light scattering measurements, the scattered part being more sensitive to surface magnetization than the reflected one. Therefore, this technique allows analyzing in-depth magnetic inhomogeneities in a simple and easy way, offering also the possibility to be used in situ.

This work was performed under Spanish CICyT financial support. One of the authors (M.U.G.) thanks the Consejería de Educación y Cultura de la Comunidad de Madrid for funding. One of the authors (C.M.B.) acknowledges a grant from the Ministerio de Ciencia y Tecnología.

${ }^{1}$ O. Geoffroy, D. Givord, Y. Otani, B. Pannetier, A.D. Santos, M. Schlenker, and Y. Souche, J. Magn. Magn. Mater. 121, 516 (1993).

${ }^{2}$ I. Guedes, N. J. Zaluzec, M. Grimsditch, V. Metlushko, P. Vavassori, B. Ilic, P. Neuzil, and R. Kumar, Phys. Rev. B 62, 11719 (2000).

${ }^{3}$ P. García-Mochales, J. L. Costa-Krämer, G. Armelles, F. Briones, D. Jaque, J. I. Martín, and J. L. Vicent, Appl. Phys. Lett. 81, 3206 (2002).

${ }^{4}$ J. M. Bennet and L. Mattson, Introduction to Surface Roughness and Scattering (Optical Society of America, Washington, DC, 1999).

${ }^{5}$ J. C. Stover, Optical Scattering: Measurement and Analysis (SPIE, Bellingham, Washington, 1995).

${ }^{6}$ M. U. González, J. A. Sánchez-Gil, Y. González, L. González, and E. R. Méndez, J. Vac. Sci. Technol. B 18, 1980 (2000).

${ }^{7}$ C. Martínez Boubeta, E. Navarro, A. Cebollada, F. Briones, F. Peiró, and A. Cornet, J. Cryst. Growth 226, 223 (2001).

${ }^{8}$ G. Armelles, J. L. Costa-Krämer, J. I. Martin, J. V. Anguita, and J. L. Vicent, Appl. Phys. Lett. 77, 2039 (2000).

${ }^{9}$ J. M. Elson, Phys. Rev. B 30, 5460 (1984).

${ }^{10}$ K. H. J. Buschow, in Ferromagnetic Materials, edited by E. P. Wohlfarth and K. H. J. Buschow (Elsevier Science, Amsterdam, The Netherlands, 1988).

${ }^{11}$ J. F. Mackay, C. Teichert, D. E. Savage, and M. G. Lagally, Phys. Rev. Lett. 77, 3925 (1996).

${ }^{12}$ C. S. Nelson, G. Srajer, J. C. Lang, C. T. Venkataraman, S. K. Sinha, H. Hashizume, N. Ishimatsu, and N. Hosoito, Phys. Rev. B 60, 12234 (1999).

${ }^{13}$ J. W. Freeland, V. Chakarian, K. Bussmann, Y. U. Idzerda, H. Wende, and C.-C. Kao, J. Appl. Phys. 83, 6290 (1998).

${ }^{14}$ J. B. Kortright and S.-K. Kim, Phys. Rev. B 62, 12216 (2000). 